University of Nebraska - Lincoln

DigitalCommons@University of Nebraska - Lincoln

6-1-1998

\title{
Strain induced alteration of the gadolinium surface state
}

C. Waldfried

University of Nebraska-Lincoln

D.N. Mcllroy

University of Nebraska-Lincoln

T. McAvoy

University of Nebraska-Lincoln

D. Welipitiya

University of Nebraska-Lincoln

Peter A. Dowben

University of Nebraska-Lincoln, pdowben@unl.edu

See next page for additional authors

Follow this and additional works at: https://digitalcommons.unl.edu/physicsdowben

Part of the Physics Commons

Waldfried, C.; Mcllroy, D.N.; McAvoy, T.; Welipitiya, D.; Dowben, Peter A.; and Vescovo, Elio, "Strain induced alteration of the gadolinium surface state" (1998). Peter Dowben Publications. 37.

https://digitalcommons.unl.edu/physicsdowben/37

This Article is brought to you for free and open access by the Research Papers in Physics and Astronomy at DigitalCommons@University of Nebraska - Lincoln. It has been accepted for inclusion in Peter Dowben Publications by an authorized administrator of DigitalCommons@University of Nebraska - Lincoln. 


\section{Authors}

C. Waldfried, D.N. Mcllroy, T. McAvoy, D. Welipitiya, Peter A. Dowben, and Elio Vescovo 


\title{
Strain induced alteration of the gadolinium surface state
}

\author{
C. Waldfried, D. N. Mcllroy, T. McAvoy, D. Welipitiya, and P. A. Dowben ${ }^{\text {a) }}$ \\ Department of Physics \& Astronomy and the Center for Materials Research and Analysis, \\ University of Nebraska-Lincoln, Lincoln, Nebraska 68588-0111 \\ E. Vescovo \\ National Synchrotron Light Source, Brookhaven National Laboratory, Upton, New York 11973
}

The electronic structure of strained and unstrained $\mathrm{Gd}(0001)$ has been studied with photoemission, inverse photoemission, and spin-polarized photoemission. A shift of the occupied majority and unoccupied minority surface states has been observed as a result of the strain, consistent with the phase accumulation model. There is a strain induced shift of the minority spin surface state across the Fermi level. (C) 1998 American Institute of Physics. [S0021-8979(98)28611-7]

Surface states may experience a shift in binding energy due to strain. ${ }^{1}$ For magnetic surfaces the spin dependent shift of the surface majority and minority subbands near the Fermi level can result in a variation of the spin population at different wave vectors $k$. This will have a substantial influence on the magnetic behavior of the surface. The subject of this article is the effect of strain on the electronic structure of the surface of gadolinium. We show consistency with the effects recently demonstrated by Neuhold and Horn $^{1}$ on the surface of $\mathrm{Ag}(111)$.

The surface magnetism of gadolinium has been the subject of controversy over the past years, mainly as a result of the proximity of the $5 d_{z^{2}-r^{2}}$, $6 s$ surface states to the Fermi level. Strain substantially alters the electronic structure of gadolinium $^{2-4}$ and is expected to result in a shift of the surface state binding energies. We have been able to obtain strained films of gadolinium, with an increased lattice constant of approximately $4 \%$ by growing gadolinium on the corrugated surface of $\mathrm{Mo}(112){ }^{2-4}$

We investigated the occupied and unoccupied electronic structure of thin films of strained and unstrained $\operatorname{Gd}(0001)$. Spin-polarized photoemission experiments were carried out at the new U5UA undulator beamline of the National Synchrotron Light Source (NSLS) at the Brookhaven National Laboratory in Upton, NY. The details of the experimental setups are described elsewhere. ${ }^{5,6}$ Inverse photoemission spectra were acquired in a different UHV system $^{2}$ in the isochromatic mode $(\hbar \omega=9.4 \mathrm{eV})$ with a Geiger-Müller tube and an electron gun based on the Zipf design. ${ }^{7}$ Strained and unstrained thin films of Gd, 10-40 ML thick, were grown by slow thermal deposition on Mo(112) and W(110) substrates, respectively. The surface and bulk character of the bands has been determined from chemisorption studies and photon energy dependence, while the symmetry of the bands has been ascertained from the light polarization dependence as described in detail elsewhere. ${ }^{2}$

The influence of strain on the surface electronic structure of $\mathrm{Gd}$ is illustrated in Fig. 1, which compares two normal emission photoemission spectra that were taken for strained and unstrained $\operatorname{Gd}(0001)$. Both spectra have been acquired

\footnotetext{
${ }^{\text {a)} E l e c t r o n i c ~ m a i l: ~ p d o w b e n @ u n l i n f o . u n l . e d u ~}$
}

with a photon energy of $33 \mathrm{eV}$ for films of similar nominal thickness of approximately $15 \mathrm{ML}$. The valence band of the strained $\mathrm{Gd}(0001)$ films is distinct from that of the relaxed films. The binding energy of the $5 d, 6 s$ strained bulk bands at $1.8 \mathrm{eV}$ below $E_{F}$ differs from the unstrained bulk bands observed at approximately 1.5 and $0.8 \mathrm{eV}$ below $E_{F}$ at the surface Brillouin zone center. More significant, the narrow surface state near the Fermi level of the unstrained Gd(0001) is shifted towards higher binding energy and appears substantially broadened.

The origin of the clearly altered valence-band states and broader density of states near the Fermi level of strained Gd is revealed by the spin resolved electronic structure, shown in Fig. 2. The unstrained Gd(0001) valence band at the Brillouin zone center $(\bar{\Gamma})$ is characterized ${ }^{8}$ by Stoner-like exchange split $5 d$ bulk bands at binding energies of approximately 1.5 (majority) and $0.8 \mathrm{eV}$ (minority) and two sets of

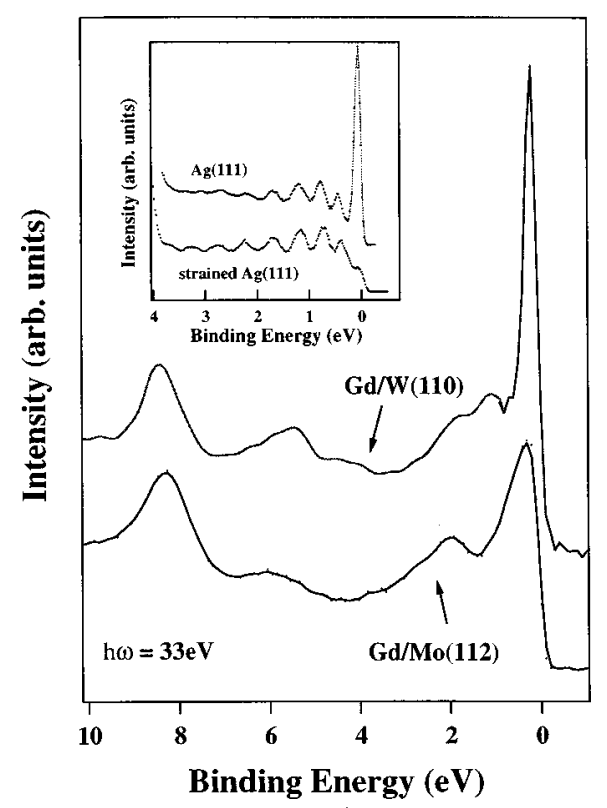

FIG. 1. Normal emission photoemission spectra for strained (bottom) and unstrained (top) films of $\mathrm{Gd}(0001)$, acquired with a photon energy of $33 \mathrm{eV}$. The inset shows a comparison of a strained (bottom) and unstrained (top) $\operatorname{Ag}(111)$ surface from Ref. 1. 


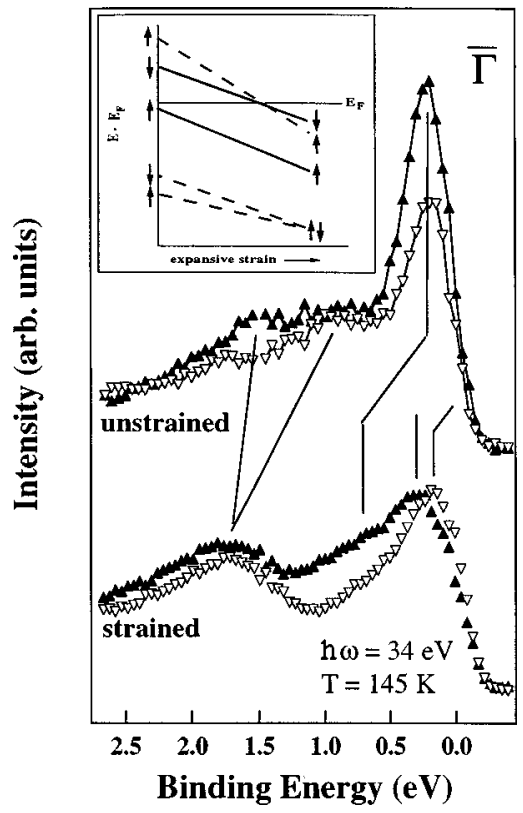

FIG. 2. Spin-polarized photoemission spectra for strained (bottom) and unstrained (top) $\mathrm{Gd}(0001)$ at normal emission and approximately $145 \mathrm{~K}$. The spectra were acquired with a photon energy of $34 \mathrm{eV}$. The inset schematically plots the binding energy shifts of the surface (solid) and bulk (dashed) spin subbands as a function of increased expansive strain.

spin majority and minority subbands of the surface on either side of the Fermi level. The occupied predominantly spinmixed majority $5 d_{z^{2}-r^{2}}$, $6 s$ surface state is located at approximately $0.1 \mathrm{eV}$ below $E_{F}$ (Fig. 2) and the unoccupied minority counter part of the surface state at approximately $0.3 \mathrm{eV}$ above the Fermi level ${ }^{9}$ (Fig. 3) (though this is dependent on temperature well away from $\left.T_{C}\right){ }^{10}$

In the strained Gd films the bulk spin majority and minority subbands are found at approximately $1.8 \mathrm{eV}$ binding energy at $\bar{\Gamma}$, with negligible Stoner-like exchange splitting. ${ }^{3}$ There are three features near the Fermi level $(0$ to $1 \mathrm{eV}$ binding energy) in the valence band of the strained Gd films. Two features, the spin majority state at $0.7 \mathrm{eV}$ below $E_{F}$ and the spin minority state at $0.2 \mathrm{eV}$ binding energy are Stoner-

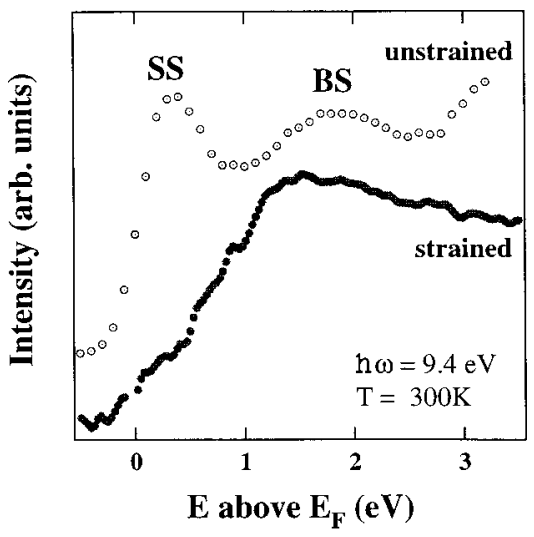

FIG. 3. The unoccupied density of states for strained (bottom) and unstrained (top) films of $\mathrm{Gd}(0001)$. The spectra were taken at approximately $300 \mathrm{~K}$. The data for the unstrained Gd has been extracted from Ref. 9. Surface and bulk features are indicated by the surface state (SS) and bulk state (BS), respectively. like exchange split surface bands. ${ }^{3}$ The surface character of these two states has been verified by their sensitivity to small amounts of adsorbates and their two dimensionality of state. Both surface sensitive features do not disperse with changing perpendicular momentum ${ }^{2}$ and are therefore confined to the two-dimensional plane at the surface. The third feature in the valence-band region near $E_{F}$, an additional bulk band of majority character, is located in the exchange splitting gap at approximately $0.4 \mathrm{eV}$ binding energy at $T / T_{C}<0.7$.

The two occupied bands closest to $E_{F}$, the spin minority surface subband, and the spin majority bulk band are newly introduced into the occupied density of states as a result of the strain. Both bands are found in the unoccupied region for the unstrained Gd films as seen in Fig. 3. Expansive strain within the Gd films induces a "downward" shift of the unoccupied surface state across the Fermi level. This is consistent with the absence of the surface spin minority state $(0.3$ $\mathrm{eV}$ above $\left.E_{F}\right)^{9}$ in the unoccupied density of states of the strained $\mathrm{Gd}$ films. The spin majority bulk band at $0.4 \mathrm{eV}$ binding energy of the strained Gd film is postulated to originate from the unstrained bulk band, located approximately $1.5 \mathrm{eV}$ above the Fermi level, ${ }^{9}$ at zone center, crossing the Fermi level about halfway across the zone in unstrained Gd. This shift in binding energy at $\bar{\Gamma}$ is illustrated in the inset of Fig. 2. More detailed studies of the occupied and unoccupied density of states are, nonetheless, necessary to confirm this postulate.

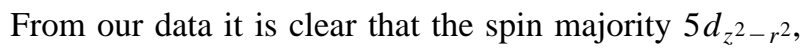
$6 s$ surface state is shifted from $0.2 \mathrm{eV}$ binding energy for the unstrained $\mathrm{Gd}$ films to $0.7 \mathrm{eV}$ in the strained $\mathrm{Gd}(0001)$. A similar downward shift of $0.5 \mathrm{eV}$ is observed for the spin minority counterpart, which is unoccupied for the "relaxed" $\mathrm{Gd}(0001)$ and found at a binding energy of approximately $0.2 \mathrm{eV}$ below $E_{F}$ due to strain (Fig. 2 inset). Concomitantly, the occupied spin minority $\left(0.8 \mathrm{eV}\right.$ below $\left.E_{F}\right)$ and unoccupied spin majority $\left(\approx 1.5 \mathrm{eV}\right.$ above $\left.E_{F}\right)$ bulk bands of unstrained Gd experience large shifts of approximately 1.0 and $1.9 \mathrm{eV}$, respectively.

The shift to higher binding energies of the Gd surface states (majority and minority) under the influence of expansive strain is in agreement with the strain induced shifts of the surface state binding energy for $\operatorname{Ag}(111){ }^{1}$, which is shown in the inset of Fig. 1. There, compressive strain results in the upward shift of the sharp $\operatorname{Ag}(111)$ surface state across the Fermi level, where it is cut off by the Fermi function and undetectable with photoemission. In both cases the strained induced shift of the surface state(s) can be explained by the phase accumulation model. ${ }^{11-14}$

Surface states originate from electrons confined to the top most layer of the solid, trapped in between the bulk band gap and the surface potential. A simple one-dimensional (socalled "phase accumulation") model ${ }^{11-14}$ can be employed to roughly describe the surface state energy. This simple model considers the surface electron in a one-dimensional quantum well, confined by the image potential on the vacuum side and the bulk band gap on the crystal side. The surface state energy is then determined by the lowest allowed quantum mechanical solution of the electron wave function. The energy of the surface state thus varies proportionally to 
$1 / d^{2}$, with $d$ being the width of the well. In the phase accumulation formalism, $d$ is dependent, among others, on the bulk band edges ${ }^{15}$ and the work function. ${ }^{11,13}$ Thus the strain induced shifts in binding energy of the surface states in gadolinium can be attributed to the altered majority and minority bulk band gaps and the reduced work function of strained $\mathrm{Gd}$, approximately $2.2 \mathrm{eV}$, as compared to $3.3 \mathrm{eV}$ for the relaxed $\mathrm{Gd}$ film.

Our results provide evidence that under expansive strain both spin components of the bulk bands are modified and results in a downward shift of the surface spin subbands. A change of the in-plane lattice constant of approximately $4 \%$ yields a surface state energy shift of $0.5 \mathrm{eV}$ (Fig. 2 inset). The strain induced shifts of the bulk band edges (spin majority and spin minority) of approximately 1 to $2 \mathrm{eV}$ are substantially larger than those of the surface bands. The shift of each Gd surface state (one in the spin majority band structure, the other in the spin minority band structure) is consistent with the observations and calculation for $\operatorname{Ag}(111){ }^{1}$

This study shows that strain can significantly alter the spin-polarized electronic structure of a ferromagnetic system like gadolinium in much the same way as strain affects a nonmagnetic system like $\mathrm{Ag}(111)$. In a ferromagnetic system, we need to consider each surface state spin component in the appropriate spin-resolved band structure. It is only apparent in the spin-resolved band structure how similar the influence of strain is in both $\operatorname{Ag}(111)$ and $\operatorname{Gd}(0001)$.

\section{ACKNOWLEDGMENTS}

This work was supported by NSF through Grant Nos. DMR-92-21655, DMR-94-96131, and DMR-94-07933. The experiments were carried out at the National Synchrotron Light Source which is funded by the DOE and at the Synchrotron Radiation Center which is also funded by NSF.

${ }^{1}$ G. Neuhold and K. Horn, Phys. Rev. Lett. 78, 1327 (1997).

${ }^{2}$ C. Waldfried, D. N. McIlroy, and P. A. Dowben, J. Phys.: Condens. Matter 9, 10615 (1997).

${ }^{3}$ C. Waldfried, D. Welipitiya, T. McAvoy, E. Vescovo, and P. A. Dowben, Phys. Rev. Lett. (submitted).

${ }^{4}$ C. Waldfried, D. N. McIlroy, and P. A. Dowben, Phys. Rev. B 54, 16460 (1996); 56, 9973 (1997).

${ }^{5}$ E. Vescovo et al., Activity Report 1996, Nat. Synch. Light Source, A-25 (1997).

${ }^{6}$ P. D. Johnson et al., Rev. Sci. Instrum. 63, 1902 (1992); J. Unguris, D. T. Pierce, and R. J. Calotta, Rev. Sci. Instrum. 57, 1314 (1986).

${ }^{7}$ P. W. Erdmann and E. C. Zipf, Rev. Sci. Instrum. 53, 225 (1982).

${ }^{8}$ D. Li, J. Pearson, S. D. Bader, D. N. McIlroy, C. Waldfried, and P. A. Dowben, Phys. Rev. B 51, 13895 (1995).

${ }^{9}$ M. Donath, B. Gubanka, and F. Passek, Phys. Rev. Lett. 77, 5138 (1996).

${ }^{10}$ M. Bode, M. Getzlaff, S. Heinze, R. Pascal, and R. Wiesendanger, Phys. Rev. A (in press).

${ }^{11}$ N. V. Smith, Phys. Rev. B 32, 3549 (1995).

${ }^{12}$ N. V. Smith, N. B. Brooks, Y. Chang, and P. D. Johnson, Phys. Rev. B 49, 332 (1994).

${ }^{13}$ P. Ahlqvist, Solid State Commun. 31, 1029 (1979).

${ }^{14}$ P. M. Echenique and J. B. Pendry, J. Phys. C 11, 2065 (1978).

${ }^{15}$ R. Paniago, R. Matzdorf, G. Meister, and A. Goldmann, Surf. Sci. 336, 113 (1995). 